\title{
JPEG Image Compression Using Discrete Cosine Transform
}

\author{
Damaraju Sri Sai Satyanarayana, Govindareddy Rahul Reddy, \\ R.S.P. Shanmukhanath, Kumar Gaurav
}

\begin{abstract}
In this paper, Baseline JPEG standard using has been implemented along with encoding and decoding of gray scale images in JPEG. The first step in encoding starts by dividing the image in $8 * 8$ blocks into sub-images on which DCT is performed which is followed by dividing the resulted matrices by a Quantization matrix. The algorithm ends by making the data one-dimensional which is done by Zigzag Coding and composed by Arithmetic coding or Huffman Coding. Reversing the process of encoding results in decoding process. At first, the received bit stream is converted back into two-dimensional matrices and multiplied by Quantization matrix and Inverse DCT is performed and the sub-images are connected to restore the image. Effect of coefficients on the image restored and difference between compression ratios has been presented in the paper.
\end{abstract}

Index Terms-JPEG, Encoding, Decoding, DCT, Quantization, Matrix.

\section{INTRODUCTION}

Multimedia images are vital and ubiquitous components of modern technology and the amount of information encoded in an image is enormous. If images are not compressed, a wide range of applications would be costly although bandwidth and storage capabilities advance. An original image requires an enormous amount of data to represent it with redundant or unnecessary information. Thus, compression is essential for storage, retrieval, and transmission of images. Compression is mainly categorized into two categories: Lossless Compression involves the original data to perfectly reconstruct from the compressed data which doesn't degrade data. This is used in cases when the original and the decompressed data be identical. Lossy Compression, on the other hand, uses partial data discarding and inexact approximations to represent the content. The amount of data reduction is much higher in lossy compression than compared to lossless.

Damaraju Sri Sai Satyanarayana, Department of Electronics and Communication Engineering, Sreyas Institute of Engineering and Technology, Hyderabad, India

Govindareddy Rahul Reddy, Department of Mechanical Engineering, Manipal Institute of Technology, Manipal, India

R. S. P. Shanmukhanath, Department of Electronics and Communication Engineering, Sreyas Institute of Engineering and Technology, Hyderabad, India

Kumar Gaurav, Department of Applied Electronic and Instrumentation Engineering, Gandhi Institute of Engineering and Technology, Orissa, India.

\section{TYPES OF IMAGE COMPRESSION}

Image Compression applied to digital images is a type of data compression, to reduce their cost for storage or transmission. Algorithms may take advantage of Statistical propertied and the visual perception to provide better results compared with general data compression methods. They are mainly categorized into two categories: Lossless and lossy.

\section{A. Lossless Compression}

Also known as Entropy coding, involves the original data to perfectly reconstruct from the compressed data which doesn't degrade data. This is used in cases when the original and the decompressed data be identical. Redundancy present in image signals is directly proportional to the amount of correlation among the data samples. These do not add noise to the signal and uses decomposition techniques to minimize redundancy.

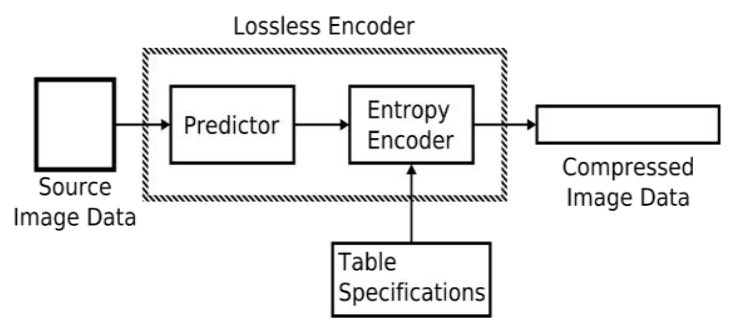

Figure 1. Lossless Compressionn Block Diagram [6]

Lossless compression includes following techniques:

1. Lempel-Ziv-Welch (LZW) Coding: This algorithm is a very common compression technique optionally used in PDF and typically used in GIF, easy to implement and has the potential for very high throughput. The idea relies on iterative patterns to save data space.

2. Huffman encoding: It is one of the basic compression methods useful for image and video compression. It is a form of statistical coding which reduces the number of bits required to represent a string.

3. Area Coding: This is an enhanced form of run-length coding which reflects the two-dimensional character of images. An array of sequences builds up a two-dimensional object for coding an image.

4. Run length encoding: For sequential data, this is a simple compression method. In this technique, identical pixels are 
replaced by shorter symbols. This scheme was employed for transmission of analog TV signals.

\section{B. Lossy Compression}

Lossy Compression on the other hand uses partial data discarding and inexact approximations to represent the content. The amount of data reduction is much higher in lossy compression than compared to lossless. Lossy method is used for most applications.

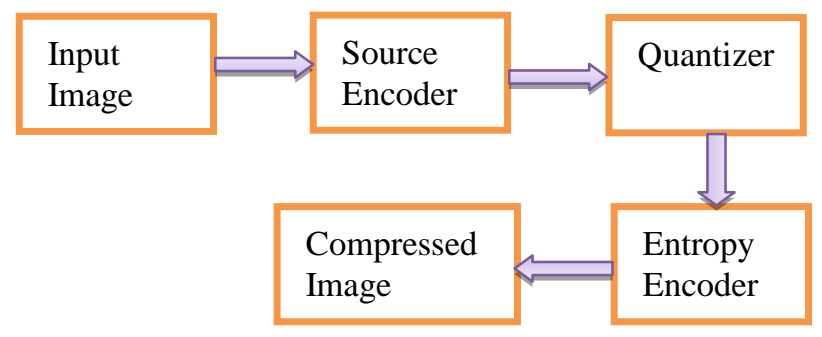

Figure 2. Lossy Compression Block Diagram

Lossy compression includes following methods:

1. Fractal Coding: It is a process to encode an image as a set of mathematical data which describes the fractal properties of an image which has intense computation. This is widely used to convert the image into fractal codes which make decoding process simpler.

2. Sub-band Coding: This is a type of coding which breaks the signal into no. of frequency bands and encodes independently. It is the core technique used in the audio compression algorithm.

3. Block Truncation Coding (BTC): BTC is a type of image compression used for greyscale images which divides the original image into blocks and then uses a quantizer to reduce the gray scale level. This technique is also been adapted to video compression.

4. Transform Coding: Transform coding is typically lossless which is used to convert spatial image pixels to transform coefficient values. When the image is decoded, the output may not be identical to the original image.

5. Code Vector Quantization: It is a quantization technique which allows the modeling of probability density functions by prototype vectors which is originally used for data compression. Each group is represented by centroid point and other by clustering algorithms

\section{IMAGE COMPRESSION MODEL}

An image compression system is illustrated in figure (3). The original image is transformed into another domain and is de-correlated by the transform which concentrates image information to impact form. The redundancy in the transformed image is removed by the encoder and stored in a data stream. To recover the image, the decoder reverses this process and the image might have distortion when compared to the original image.

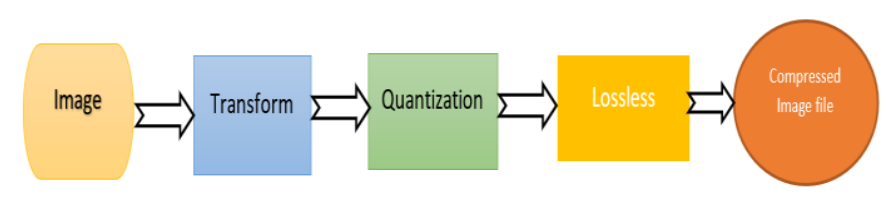

Figure 3. Image Compression Model

\subsection{Transform}

Transform is the first stage in the compression model which converts the data from a spatial domain into the frequency domain. Discrete Cosine Transform (DCT) is used as a technique for conversion. A DCT expresses a finite sequence of data in the form of cosine functions oscillating at different frequencies. Significant coefficients are produced by DCT which leads to greater compression. The most delayed part of JPEG Compression is computing DCT.

Two-Dimensional Discrete Cosine Transform: [2]

Speech waveforms are processed using one-dimensional DCT. For image analysis, two-dimensional DCT is needed. Applying one-dimensional transforms separately to rows and columns, two-dimensional DCT is computed. The Discrete cosine transform of real numbers is given by:

$$
\begin{aligned}
& S(u, v)=\underset{n}{\left(-\frac{-1}{2}\right.} C(u) C(v) \sum_{x=0}^{n-1} \sum_{y=0}^{m-1} s(x, y) \cos \left(\frac{(2 x+1) u \pi}{2 n}\right) \cos \left(\frac{(2 x+1) v \pi}{2 m}\right) \text { for } \mathrm{u}=0 \ldots \mathrm{n}-1, \\
& C(u), C(v)=\left\{\begin{array}{lc}
\frac{-1}{2} & \text { for } u, v=0 \\
1 & \text { otherwise }
\end{array}\right. \\
& \mathrm{v}=0 \ldots \mathrm{m}-1
\end{aligned}
$$

Inverse Discrete Cosine Transform is given by:

$$
\begin{aligned}
& s(x, y)=\underset{n}{(-)}{ }_{n=0 v=0}^{\frac{-1}{2}} \underset{\sum}{\sum} \underset{i m-1}{\sum}(u) C(v) S(u, v) \cos \left(\frac{(2 x+1) u \pi}{2 n}\right) \cos \left(\frac{(2 x+1) v \pi}{2 m}\right) \text { for } \mathrm{x}=0 \ldots \mathrm{n}-1, \\
& \text { where } C(u), C(v)= \begin{cases}\frac{-1}{2} & \mathrm{y}=0 \ldots \mathrm{m}-1 \\
2^{2} & \text { for } u, v=0\end{cases}
\end{aligned}
$$

\subsection{Quantization}

After FDCT, 64 DCT coefficients are uniformly quantized in conjunction with Quantization table. Quantization is used for further compression using DCT Coefficients to achieve the desired image quality. Quantization aims to reduce high-frequencyDCT coefficients to zero and low frequencies to reconstruct the image.

$$
\begin{gathered}
F(u, v)_{\text {Quantization }}=\text { round }\left(\frac{F(u, v)}{Q(u, v)}\right) \\
F(u, v)_{d e Q}=F(u, v) \text { Quantization } \times Q(u, v)
\end{gathered}
$$




\subsection{Zig-Zag Sequence}

Zig-Zag scanning is a transform-based scanning employed for non-uniform quantization of $\mathrm{n} \times \mathrm{n}$ matrix DCT coefficient. After Quantization, all the DC coefficients are placed into Zig-Zag sequence as shown in figure 4 which helps to expedite entropy coding by sequencing low-frequency coefficients before high-frequency coefficients.

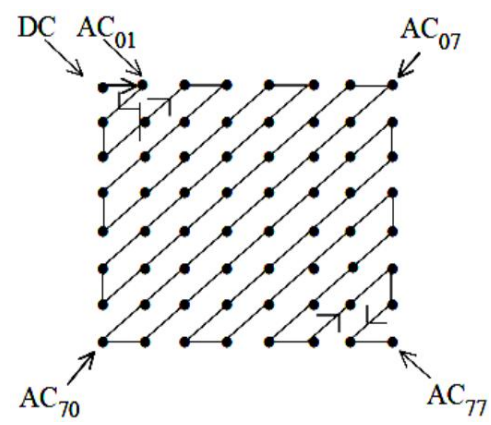

Figure 4. ZigZag Sequencing [2]

\subsection{Entropy Coding}

Arithmetic coding and Huffman coding are the two entropy coding methods specified in JPEG proposal. The first step converts the quantized sequence into an intermediate sequence of symbols. The second step converts symbols to a data stream in which symbols no longer have boundaries. Arithmetic coding has produced a better comparison than Huffman coding for images.

The entire DCT procedure is shown in figure 5:

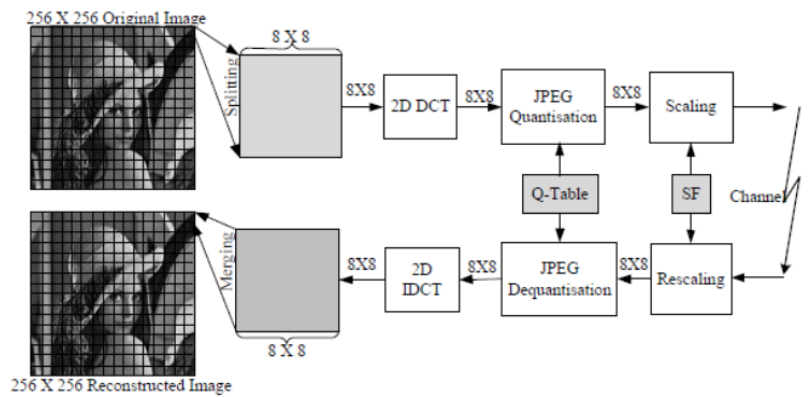

Figure 5. Block Diagram of the JPEG based DCT

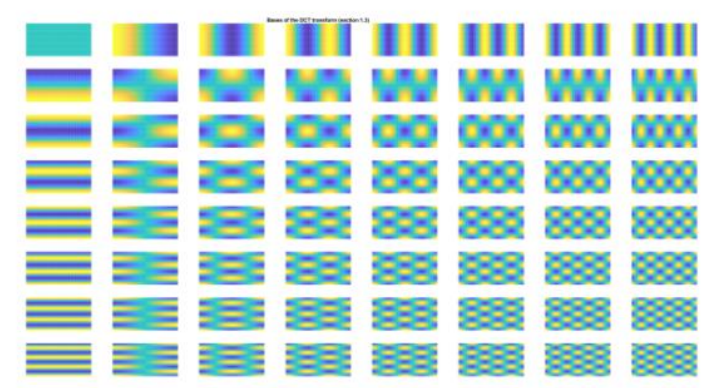

Figure 7. The (8x8) array of basis images for the (2-D) DCT
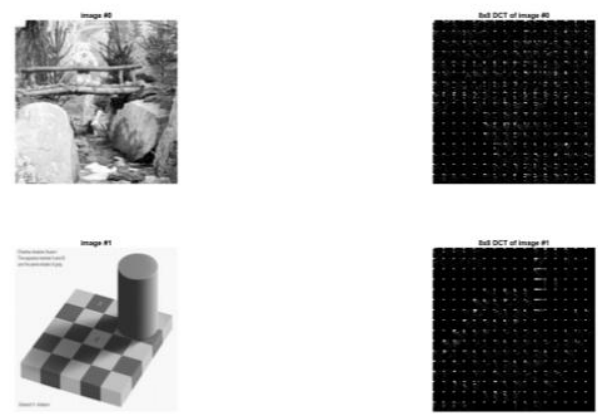

Figure 8. $8 \times 8$ DCT of Image
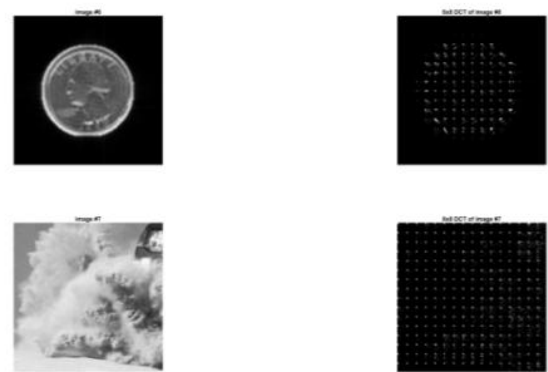

Figure 9. $8 \times 8$ DCT of Image

IV. RESULTS

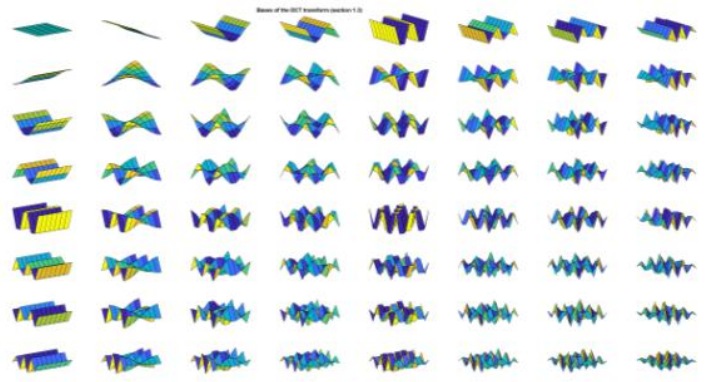

Figure 6. Bases of the DCT Transform

Figure 10. Power of DCT Coeffecients 

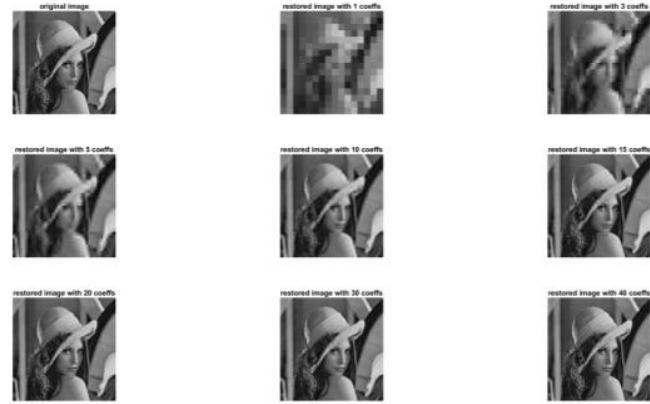

Figure 11. Restored Image with Coeffecient factor

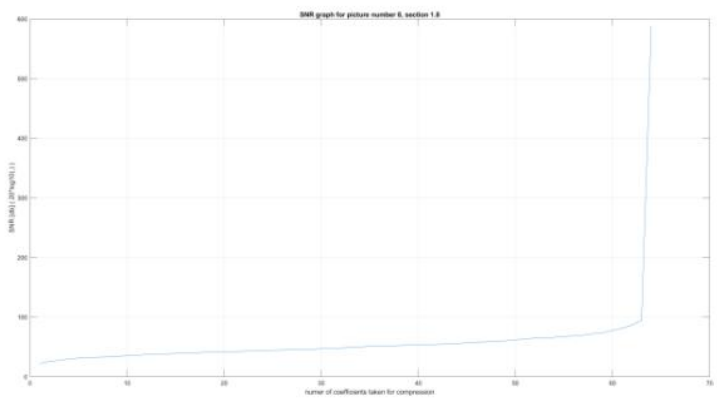

Figure 12. SNR Graph of Image

\section{ADVANTAGES AND DISADVANTAGES OF IMAGE COMPRESSION}

\section{A. Advantages}

1. Size of JPEG images can be compressed and decreased which makes the document size reasonable for transferring images over the internet since it devours less data transfer capacity.

2. JPEG format can be used to store high-resolution images and can be stored quickly in a storage device.

3. Format of Image is compatible with most of the hardware components.

4. Since fewer bits are transferred, it reduces the transmission errors.

\section{B. Dis-Advantages}

1. JPEG Images do not support layered images.

2. Image compression is not suitable for pictures with sharp edges and lines.

\section{CONCLUSION}

The JPEG algorithm was designed to compress images and the results depict high compression ratios and allow a user to choose the quality of the output image. The algorithm compresses the images in 4 different phases using DCT. The effect of coefficients on image restoration is shown.

\section{REFERENCES}

[1] Beenish Khan, Ms. Poonam, Mr. Mohammad Talib "Matlab Based Image Compression Using Various Algorithm" Published in International Journal of Trend in Scientific Research and Development (ijtsrd), ISSN: 2456-6470, Volume-2 | Issue-4, June 2018, pp.1638-1642.

[2] Kadhim, Qusay Kanaan. "Image compression using Discrete Cosine Transform method." Int. J. Comput. Sci. Mob. Comput. (2016): 186.

[3] Rojatkar, Dinesh V., et al. "Image Compression Techniques: Lossy and Lossless." International Journal of Engineering Research and General Science 3.2 (2015).

[4] Raid, A. M., et al. "Jpeg image compression using discrete cosine transform-A survey." arXiv preprint arXiv: 1405.6147 (2014).

[5] More, Nitesh Kumar, and Sipi Dubey. "JPEG Picture Compression Using Discrete Cosine Transform." International Journal of Science and Research (IJSR) 2.1 (2013): 134-138.

[6] Sindhu, M., and R. Rajkamal. "Images and its compression techniques-A Review." International Journal of Recent Trends in Engineering 2.4 (2009): 71.

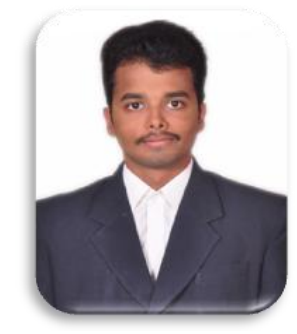

Damaraju Sri Sai Satyanarayana obtained his B.Tech degree in Electronics and Communication engineering from Sreyas Institute of Engineering and Technology. He has co-authored in 4 publications mainly in antenna. His current research focuses on Image processing. His area of research interests are Antenna design, Signal Processing, Telecommunications and Image processing.

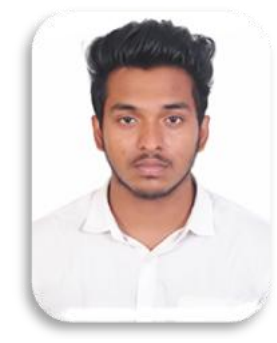

Govindareddy Rahul Reddyis a fourth-year Mechanical engineer student at Manipal Institute of Technology about to receive a bachelor's degree in 2020. His research interests include suspension systems, material composites, marketing research. Rahul can be contacted at rahulreddy19.07@gmail.com

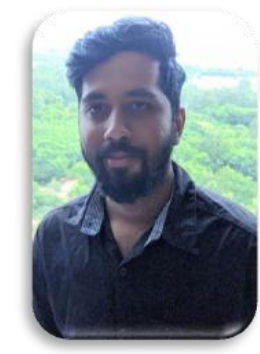

R.S.P.Shanmukhanath earned his bachelor's degree from Sreyas Institute of Engineering and Technology in Electronics and Communication specialization. He was also the cinematographer for short films directed by Sarcasm films. His present area of interest is Image processing and Communication engineering. 